\title{
Factores asociados con dispepsia funcional en trabajadores de establecimientos de comida rápida en un centro comercial de Huancayo, Perú
}

\author{
Factors associated with functional dyspepsia among fast food workers at a \\ shopping center in Huancayo, Peru
}

Jermaine Ramírez-Vásquez, MS, ${ }^{1}$ Christian R. Mejia, MD, MSc, PhD. ${ }^{2}$

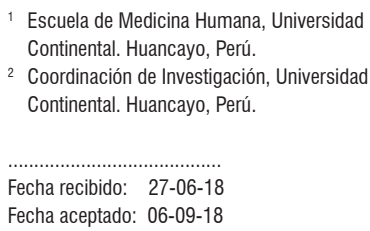

\begin{abstract}
Resumen
Introducción: La dispepsia funcional (DF) comprende trastornos crónicos y recurrentes del tracto digestivo superior y no tiene origen orgánico. Objetivos: Determinar los factores asociados con DF en trabajadores de establecimientos de comida rápida en un centro comercial de Huancayo, Perú. Metodología: Estudio transversal analítico, se encuestó a 128 trabajadores de establecimientos de comida rápida en una ciudad de la serranía peruana. Se usó la prueba "A new questionnaire for the diagnosis of dyspepsia" para el diagnóstico de DF, esto se cruzó con variables sociales, de consumo y de hábitos alimenticios; se obtuvieron estadísticas de asociación. Resultados: El 40 \% tuvo DF. Estuvo asociado con una menor frecuencia de DF el sexo femenino (razones de prevalencias ajustadas [RPa]: 0,64; intervalo de confianza [IC] $95 \%$ : 0,41-0,99; valor $p=0,044$ ); en cambio, los que tenían dificultad para conciliar el sueño o padecían de despertares nocturnos (RPa: 1,89; IC $95 \%: 1,37-2,61$; valor $p<0,001$ ) y los que tomaban alcohol regularmente tenían mayor frecuencia de DF (RPa: 3,28; IC 95 \%: 1,02-10,6; valor $p=0,047$ ); ajustadas por 5 variables. Según los hábitos de alimentación, se encontró que estuvo asociado con una mayor frecuencia de dispepsia quien omitiera comer el almuerzo (RPa: 1,88; IC $95 \%$ : 1,22-2,90; valor $p=0,004$ ), quien omitiera la cena (RPa: 3,44; IC 95 $\%: 2,72-4,35$; valor $p<0,001)$ y quien se alimentase regularmente en otros restaurantes (RPa: 1,87 ; IC $95 \%$ : $1,22-2,88$; valor $p=0,004$ ); ajustadas por 3 variables. Conclusiones: se encontraron algunas características asociadas con la DF en esta población que comparte características con otras similares, lo que puede ser útil para generar estrategias de detección y diagnóstico oportunos.
\end{abstract}

Palabras clave

Dispepsia funcional, hábitos alimenticios, gastritis, estrés, comida rápida, salud laboral, Perú.

\begin{abstract}
Introduction: Functional dyspepsia includes chronic and recurrent disorders of the upper digestive tract and which have no organic origin. Objectives: The objectives of this study were to determine factors associated with functional dyspepsia among the workers of fast food establishments at a mall in Huancayo, Perú. Methodology: This is an analytical cross-sectional study of 128 fast food workers in a city in the Peruvian highlands. "A new questionnaire for the diagnosis of dyspepsia" was used to diagnose functional dyspepsia and record social variables, consumption and eating habits. From this information statistical measures of association were obtained. Results: Forty percent of those surveyed had functional dyspepsia. There was a lower frequency of functional dyspepsia among women than men (Prevalence ratio: 0.64, 95\% Cl: 0.41-0.99, $\mathrm{p}$-value $=0.044$ ). Those who had difficulty falling asleep and/or suffered from nighttime awakening (Prevalence ratio: $1.89,95 \% \mathrm{Cl}: 1.37-2.61, \mathrm{p}$ value $<0.001$ ) and those who drank alcohol regularly (Prevalence ratio: 3.28 , $95 \% \mathrm{Cl}: 1,02-10.6, \mathrm{p}$ value $=0.047$ ) had a higher frequency of functional dyspepsia. This was adjusted by five variables. According to eating habits, it was found that a higher frequency of dyspepsia was associated with skipping lunch (Prevalence ratio: $1.88,95 \% \mathrm{Cl}$ : 1.22-2.90, p-value $=0.004$ ), skipping dinner (Prevalence ratio: $3.44,95 \% \mathrm{Cl}: 2.72-4.35$, p value $<0.001$ ) and eating regularly in other restaurants (Prevalence ratio: $1.87,95 \%$ $\mathrm{Cl}: 1.22-2.88 ; p=0.004)$. This was adjusted by three variables. Conclusions: We found some characteristics associated with functional dyspepsia in this population shares characteristics with similar populations which could be useful for developing strategies for detection and timely diagnosis.
\end{abstract}

Keywords

Functional dyspepsia, eating habits, gastritis, stress, fast food, occupational health, Peru. 


\section{INTRODUCCIÓN}

La dispepsia funcional (DF) es considerada una enfermedad de naturaleza multifactorial y de gran influencia biopsicosocial (1). Sin embargo, existen algunas poblaciones laborales que podrían tener una mayor exposición y/o padecimiento de la misma, y uno de estos grupos es el personal que labora en establecimientos de comida rápida, ya que, debido a la gran demanda de estos establecimientos, dichos trabajadores están sujetos a influencias psicosociales; cambios de estilos de vida; presión laboral; adicciones (como el alcohol, tabaco y otras drogas), trastornos de ansiedad, depresión, estrés e irritabilidad (2). Todos estos factores son los que más se han asociado con el padecimiento de enfermedades gastrointestinales, como la DF (3).

Es importante medir esto por la gran variación que puede tener esta enfermedad, se han reportado prevalencias entre el 11 \% y 29 \% (4). En América Latina se encontraron estudios en Brasilia, donde la prevalencia de DF fue del $25 \%$ (5). En Perú, los estudios de esta enfermedad se han realizado en la población de la Selva, donde se encontró una prevalencia de $38 \%$, y estuvo asociada con hábitos alimenticios (6). Asimismo, se han encontrado estudios similares, cuya prevalencia de DF en trabajadores de salud fue del 32 $\%$ y los principales factores fueron la ansiedad y la depresión (7). En Huancayo, Perú, todavía no hay estudios para determinar los diferentes factores asociados con DF.

Debido a esto, nuestro objetivo fue determinar los factores asociados con DF en trabajadores de establecimientos de comida rápida en un centro comercial de Huancayo, Junín.

\section{METODOLOGÍA}

Se realizó un estudio de tipo analítico transversal que se basó en la aplicación de una encuesta a los trabajadores de empresas que expenden comida rápida, esto se realizó en un centro comercial ubicado en la ciudad de Huancayo, Perú. Dicha población comparte características similares con otras ya que son en su mayoría jóvenes y adultos jóvenes, que laboran como único medio de ingreso económico y que en su mayoría realizan estudios de nivel superior.

Para la realización de la investigación primero se generó el proyecto, que fue evaluado y aprobado por el comité de ética del Hospital San Bartolomé en Lima, el cual cuenta con el aval del máximo ente de regulación de ética en la investigación en Perú: el Instituto Nacional de Salud de Perú.

Después de la aprobación del proyecto, se solicitó al centro comercial y a cada institución el permiso para realizar la recolección de datos, esto fue mediante la conversación con las autoridades correspondientes, se les explicó el objetivo de la misma y que las encuestas no afectarían el trabajo de sus colaboradores. Luego de obtener los permisos se pidió el acceso a cada establecimiento de comida rápida para realizar la encuesta a cada trabajador, esto se realizó en los momentos libres de cada empleado o durante el receso de las capacitaciones semanales que les brindaban, con esto se aseguró que ellos pudieran resolver las encuestas en un ambiente adecuado y con la tranquilidad del caso.

Se excluyó a los trabajadores con el diagnóstico de enfermedad por reflujo gastroesofágico, gastritis, enfermedad por úlcera péptica y las encuestas con datos incompletos en relación con nuestra principal variable (menos del $1 \%$ ).

Nuestra principal variable fue la DF, calculada por la prueba validada en español, "A new questionnaire for the diagnosis of dyspepsia" (8), ya que tiene una alta sensibilidad (95\%) y especificidad (100\%). Este cuestionario consta de 9 ítems y se consideró positivo a quienes obtuvieron un puntaje $\geq 3$. Las variables independientes evaluadas fueron los factores socioeconómicos (sexo, edad, estado civil, paternidad, independencia económica, problemas para conciliar el sueño); el nivel académico (estudios universitarios, si actualmente estudia); medidas antropométricas y los antecedentes clínicos; índice de masa corporal (IMC; según los criterios de la Organización Mundial de la Salud [OMS]); los hábitos alimenticios (lugar donde se alimenta frecuentemente, cumplimiento del horario de alimentación, cantidad de alimentos que consume); y los hábitos nocivos como el tabaco (escala de Fagerström, se consideró positivo a quienes consiguieron 4 de 10 puntos) (9), el alcohol (escala de CAGE, se consideró positivo a quienes consiguieron 2 de 4 puntos) (10), el consumo de café y el consumo de bebidas energizantes.

Luego de la obtención de las encuestas, se pasó dicha información al programa Microsoft Excel, es en este paso que se realizó el control de la calidad de los datos. Luego se exportó la base al programa estadístico Stata, en su versión 12,0. En dicho programa se ejecutó el análisis descriptivo de las variables cualitativas (para lo cual se usaron las frecuencias y porcentajes) y el análisis descriptivo de las variables cuantitativas (previo al análisis de estas variables se realizó la evaluación de la normalidad mediante el uso de la prueba estadística "Shapiro Wilk", con esto se determinó que se debía describir con las medianas y rangos intercuartílicos). Después se calculó el Alpha de Cronbach de la encuesta $(0,85)$, que demostró tener gran homogeneidad de las respuestas. También se calculó la potencia estadística del cruce de las variables, casi todas tuvieron una potencia superior al $80 \%$ (solo para el cruce con el consumo de tabaco tuvo una potencia del $40 \%$, por lo que los resultados de este cruce serán netamente referenciales).

Por último, se realizó la estadística analítica, en la cual se usaron modelos lineales generalizados, con la familia Poisson, función de enlace log, modelos robustos y ajusta- 
dos por el lugar de trabajo; con esto se obtuvieron las razones de prevalencia (RP), los intervalos de confianza del 95 $\%$ (IC $95 \%$ ) y los valores $p$. En esta etapa se usó un nivel de confianza del $95 \%$ y se consideraron los valores $p<0,05$ como estadísticamente significativos.

\section{RESULTADOS}

Se encuestó a la totalidad de las 128 personas que laboran en estos establecimientos de comida rápida. El 63,3\% (81) fue de sexo masculino, la mediana de edades fue 21 años (rango intercuartílico: 20-23 años); el 86,7 \% (111) no tenía pareja, el 84,4 \% (108) no tenía hijos, la mediana del IMC fue 23 (rango intercuartílico: 22-25), el 75,0\% (96) estudiaba en la universidad, el 56,3\% (72) no tenía dificultad para conciliar el sueño y/o padecía de despertares nocturnos, el 78,1 \% (100) respetaba sus horarios de alimentación, el 57,0 \% (73) se alimentaba en su casa, el 87,5 \% (112) consumía alcohol, el 77,3 \% (99) consumía tabaco, el 78,9 \% (101) consumía café, el 53,1 \% (68) consumía bebidas energizantes, el 38,3 \% (49) omitía tomar desayuno, el 64,8 \% (83) omitía comer almuerzo y el 42,2 \% (54) omitía cenar. Por último, el 39,8 \% (51) presentó $\mathrm{DF}$, en comparación con el 60,2 \% (77), que no lo presentó (Tabla 1).

Según los síntomas con mucha frecuencia (valores en amarillo en la Figura 1), el síntoma más común fue la sensación de ardor (20\%), seguida por la pesadez estomacal (11\%) y el dolor que calma al comer (11\%) (Figura 1).
Tabla 1. Características de los trabajadores de puestos de comida rápida en Huancayo, Perú

\begin{tabular}{lcc}
\hline \multicolumn{1}{c}{ Variable } & Frecuencia & Porcentaje \\
\hline Sexo masculino & 81 & 63,3 \\
Edad (años) $^{*}$ & 21 & $20-23$ \\
Sin pareja sentimental & 111 & 86,7 \\
Sin hijos & 108 & 84,4 \\
IMC $^{*}$ & 23 & $22-25$ \\
Estaba en la universidad & 96 & 75,0 \\
Padecía de insomnio & 72 & 56,3 \\
Respetaba sus horarios de comer & 100 & 78,1 \\
Se alimentaba en casa & 73 & 57,0 \\
Se alimentaba en el trabajo & 31 & 24,2 \\
Se alimentaba en otros restaurantes & 24 & 18,0 \\
Consumía alcohol & 112 & 87,5 \\
Consumía tabaco & 99 & 77,3 \\
Consumía café & 101 & 78,9 \\
Consumía bebidas energizantes & 68 & 53,1 \\
Omitía tomar desayuno & 49 & 38,3 \\
Omitía comer almuerzo & 83 & 64,8 \\
Omitía comer cena & 54 & 42,2 \\
Con DF & 51 & 39,8 \\
Sin DF & 77 & 60,2 \\
\hline
\end{tabular}

${ }^{*}$ Se presentan las medianas y rangos intercuartílicos.

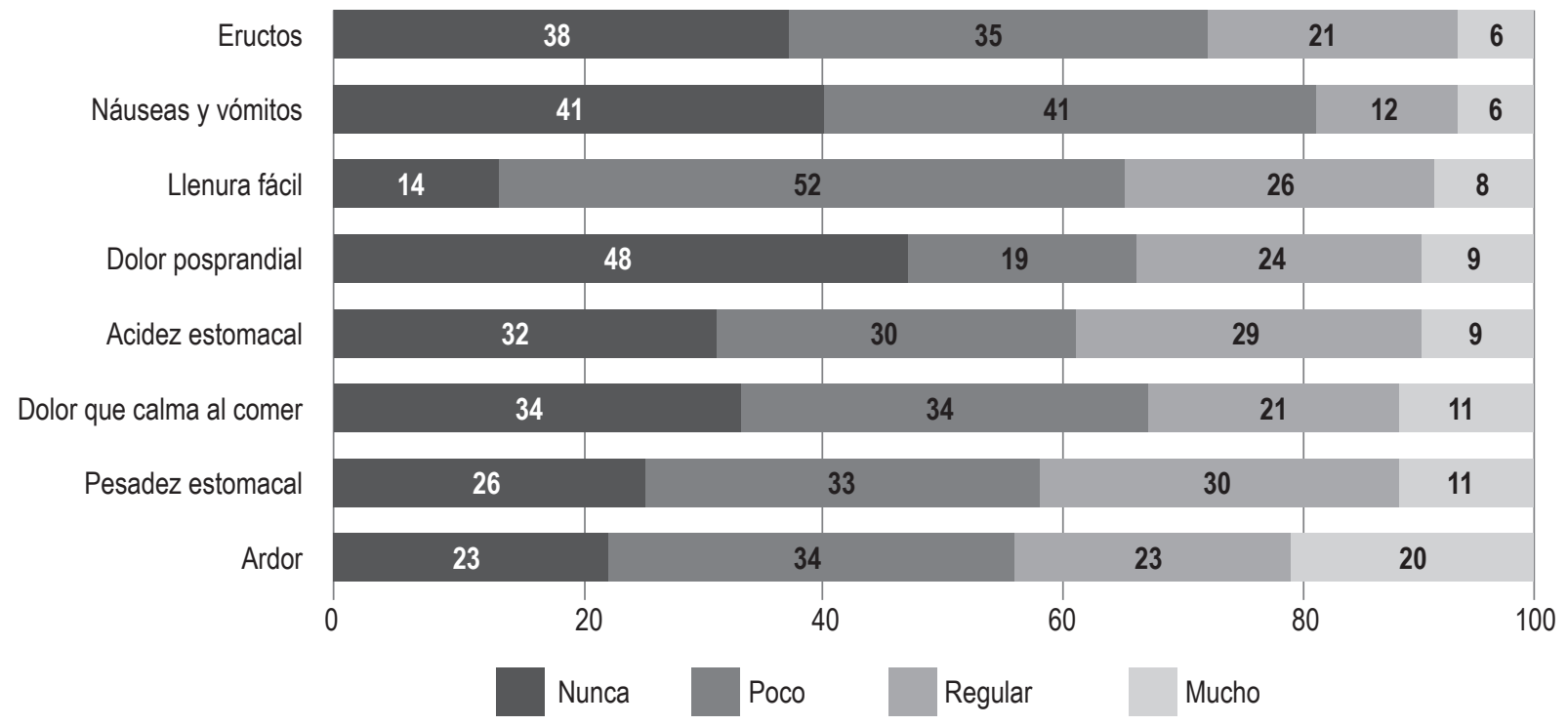

Figura 1. Porcentajes de los síntomas evaluados por la escala de DF entre los trabajadores de puestos de comida rápida en Huancayo, Perú. 
En la Tabla 2 se muestra que la DF estuvo asociada con una menor frecuencia en el sexo femenino (RPa: 0,64; IC $95 \%: 0,41-0,99$; valor $p=0,044)$; en cambio, los que tenían dificultad para conciliar el sueño o padecer de despertares nocturnos (RPa: 1,89; IC $95 \%$ : 1,37-2,61; valor $p<0,001$ ) y los que tomaban alcohol regularmente tenían mayor frecuencia de DF (RPa: 3,28; IC $95 \%$ : 1,02-10,6; valor $p=$ $0,047)$; estos cruces fueron ajustados por tomar bebidas energizantes, tener pareja, estudiar en la universidad, el valor del IMC y la sede de trabajo (Tabla 2).

Según los hábitos de alimentación, se encontró que estuvo asociado con una mayor frecuencia de dispepsia el que omitiera comer el almuerzo (RPa: 1,88; IC $95 \%$ : 1,222,90 ; valor $p=0,004$ ), que omitiera la cena (RPa: 3,44 ; IC
$95 \%: 2,72,4,35$; valor $p<0,001)$ y que se alimentase regularmente en otros restaurantes (RPa: 1,87; IC $95 \%$ : 1,22$2,88$; valor $p=0,004)$; estas variables se ajustaron por el hecho de si respetaban sus horarios de comida, si omitían tomar el desayuno y la sede de trabajo (Tabla 3).

\section{DISCUSIÓN}

Debido a la alta prevalencia de esta patología y a no tener un origen orgánico a tratar, es muy importante identificar los factores que intervienen en la DF. Por esta razón, se evalúan las variables más relevantes que se relacionan con esta enfermedad (11), medidas en trabajadores de establecimientos de comida rápida.

Tabla 2. Asociación de la DF según variables sociales, laborales y de consumo entre trabajadores de puestos de comida rápida en Huancayo, Perú

\begin{tabular}{|c|c|c|c|c|}
\hline \multirow[t]{2}{*}{ Variable } & \multicolumn{2}{|c|}{ DF n (\%) } & \multicolumn{2}{|c|}{ Estadística analítica RP (IC $95 \%$ ) } \\
\hline & Sí & No & Bivariada & Multivariada \\
\hline \multicolumn{5}{|l|}{ Sexo } \\
\hline Femenino & $11(23,4)$ & $36(76,6)$ & $0,47(0,25-0,89)^{*}$ & $0,64(0,41-0,99)^{*}$ \\
\hline Masculino & $40(49,4)$ & $41(50,6)$ & & \\
\hline \multicolumn{5}{|l|}{ Padece de insomnio } \\
\hline Sí & $39(54,2)$ & $33(45,8)$ & $2,53(1,91-3,35) £$ & $1,89(1,37-2,61) £$ \\
\hline No & $12(21,4)$ & $44(78,6)$ & & \\
\hline \multicolumn{5}{|l|}{ Toma alcohol } \\
\hline Sí & $49(43,8)$ & $63(56,2)$ & $3,50(1,04-11,7)^{*}$ & $3,28(1,02-10,6)^{*}$ \\
\hline No & $2(12,5)$ & $14(87,5)$ & & \\
\hline \multicolumn{5}{|c|}{ Toma bebidas energizantes } \\
\hline Sí & $39(57,4)$ & $29(42,6)$ & $2,87(1,42-5,79)^{*}$ & $1,37(0,82-2,29)$ \\
\hline No & $12(20,0)$ & $48(80,0)$ & & \\
\hline \multicolumn{5}{|l|}{ Tiene pareja } \\
\hline Sí & $2(11,8)$ & $15(88,2)$ & $0,27(0,09-0,81)^{*}$ & $0,49(0,18-1,29)$ \\
\hline No & $49(44,1)$ & $62(55,9)$ & & \\
\hline \multicolumn{5}{|l|}{ Estudia en la universidad } \\
\hline Sí & $43(44,8)$ & $53(55,2)$ & $1,79(1,03-3,11)^{*}$ & $1,25(0,73-2,13)$ \\
\hline No & $8(25,0)$ & $24(75,0)$ & & \\
\hline Valor del IMC & $23(21-24)$ & $24(22-25)$ & $0,90(0,87-0,93) £$ & $0,97(0,89-1,04)$ \\
\hline Edad & $22(18-23)$ & $21(20-23)$ & $0,94(0,86-1,03)$ & No entró al modelo \\
\hline Cantidad de hijos & $0(0-0)$ & $0(0-0)$ & $0,25(0,04-1,53)$ & No entró al modelo \\
\hline \multicolumn{5}{|l|}{ Consume tabaco } \\
\hline Sí & $42(42,4)$ & $57(57,6)$ & $1,37(0,60-3,13)$ & No entró al modelo \\
\hline No & $9(31,0)$ & $20(69,0)$ & & \\
\hline \multicolumn{5}{|l|}{ Consume café } \\
\hline Sí & $44(43,6)$ & $57(56,4)$ & $1,68(0,77-3,68)$ & No entró al modelo \\
\hline No & $7(25,9)$ & $20(74,1)$ & & \\
\hline
\end{tabular}

*Valor $p<0,05 . £$ Valor $p<0,001$. Las RP, los IC $95 \%$ y los valores $p$ se obtuvieron con los modelos lineales generalizados, con la familia Poisson, función de enlace log, modelos robustos y ajustados por el lugar de trabajo. El IMC, la edad y la cantidad de hijos se analizaron como variables cuantitativas. 
Tabla 3. Asociación de la DF según los hábitos de alimentación entre trabajadores de puestos de comida rápida en Huancayo, Perú

\begin{tabular}{|c|c|c|c|c|}
\hline \multirow[t]{2}{*}{ Variable } & \multicolumn{2}{|c|}{ DF n (\%) } & \multicolumn{2}{|c|}{ Estadística analítica RP (IC $95 \%$ ) } \\
\hline & Sí & No & Bivariada & Multivariada \\
\hline \multicolumn{5}{|l|}{ Omitía comer almuerzo } \\
\hline Sí & $43(51,8)$ & $40(48,2)$ & $2,91(1,99-4,26) £$ & $1,88(1,22-2,90)^{*}$ \\
\hline No & $8(17,8)$ & $37(82,2)$ & & \\
\hline \multicolumn{5}{|l|}{ Omitía comer cena } \\
\hline Sí & $38(70,4)$ & $16(29,6)$ & $4,01(3,20-5,01) £$ & $3,44(2,72-4,35) £$ \\
\hline No & $13(17,6)$ & $61(82,4)$ & & \\
\hline \multicolumn{5}{|l|}{ Lugar donde se alimentaba } \\
\hline En casa & $29(39,7)$ & $44(60,3)$ & Cat. de comparación & Cat. de comparación \\
\hline En el trabajo & $9(29,0)$ & $22(71,0)$ & $0,73(0,60-0,90)^{*}$ & $1,78(0,98-3,25)$ \\
\hline Otros restaurantes & $13(54,2)$ & $11(45,8)$ & $1,36(1,11-1,67)^{*}$ & $1,87(1,22-2,88)^{\star}$ \\
\hline \multicolumn{5}{|c|}{ Respetaba los horarios de la comida } \\
\hline No & $18(64,3)$ & $10(35,7)$ & $1,95(1,23-3,09)^{*}$ & $1,27(0,52-3,09)$ \\
\hline Sí & $33(33,0)$ & $67(67,0)$ & & \\
\hline \multicolumn{5}{|l|}{ Omitía tomar el desayuno } \\
\hline Sí & $25(51,0)$ & $24(49,0)$ & $1,55(1,24-1,93) £$ & $1,14(0,68-1,90)$ \\
\hline No & $26(32,9)$ & $53(67,1)$ & & \\
\hline
\end{tabular}

*Valor $p<0,05 . £$ Valor $\mathrm{p}<0,001$. La RP, los IC $95 \%$ y los valores $p$ se obtuvieron con los modelos lineales generalizados, con la familia Poisson, función de enlace log, modelos robustos y ajustados por el lugar de trabajo.

Se encontró que 4 de cada 10 trabajadores presentaron DF; haciendo una prevalencia del $39,8 \%$, esta cifra es mayor en comparación con 2 estudios similares, uno realizado en Piura, en el que el $32 \%$ de trabajadores de un hospital presentó DF (1); y el otro en Lima, en el que se observó una prevalencia del $37,2 \%$ en militares (12). Esto se debe a que dichos estudios se evaluaron en otro tipo de trabajadores, con diferentes formas de vida y otros factores de riesgo, lo que generaría diferencias al momento de evaluar los factores asociados con esta enfermedad y su prevalencia.

También se encontró que el sexo femenino tiene una menor frecuencia de DF, a diferencia de otros estudios, en los que se observa que el sexo femenino tiene una mayor frecuencia de DF (13-15). Sería necesario realizar estudios más profundos que traten de indagar por qué los varones tienen esta patología, lo que puede deberse a características propias de esta población.

También se encontraron otros factores que tienen mayor asociación con la DF y se identificó que los trabajadores que tuvieron problemas para conciliar el sueño o despertares nocturnos obtuvieron mayor frecuencia de dispepsia; esto es afirmado por 2 estudios, uno realizado en Estados Unidos, donde la DF está asociada con los trastornos del sueño (16) y otra investigación en China, donde se reafirma esto encontrado (14).
Otra importante asociación que se puede observar es con el consumo de alcohol, que incrementa la frecuencia de dispepsia; esto puede explicarse por el grupo etario estudiado ya que, en su mayoría, eran jóvenes que tenían entre 20 a 23 años y son más vulnerables a este tipo de sustancias, como lo confirma un estudio realizado en Chile, donde el consumo de alcohol aumenta el riesgo de dispepsia (17). Esta información es muy preocupante, ya que, su alto consumo aumenta el riesgo de tener problemas en su salud a futuro, así como podría ocasionarles inconvenientes laborales, académicos, familiares, sociales, entre otros.

En cuanto al lugar de alimentación, se encontró que los trabajadores que se alimentaban en otros restaurantes tenían mayor riesgo de presentar dispepsia, esto puede ser explicado por la alta demanda de estos establecimientos de comida rápida, sobre todo en las horas de almuerzo y cena, y donde la mayoría de encuestados labora casi todo el día -y no dispone de un horario fijo de alimentación-, salvo en los momentos donde hay poca clientela, y donde les brindan reducido tiempo para poder comer -la mayoría de veces en horarios variables e inadecuados-, como lo explica un estudio hecho en el Perú, en el que se identificó que comer fuera de los horarios habituales aumenta la frecuencia de esta enfermedad (18). Debido a lo mencionado, estos trabajadores buscan comer en lugares cercanos a su centro de 
trabajo y donde se preparen, se sirvan y se consuman de forma rápida los alimentos, como son los otros restaurantes de comida rápida del centro comercial o comedores ambulantes adyacentes. Por tanto, al alimentarse frecuentemente en estos lugares, están más propensos a padecer dispepsia; esto concuerda con una investigación realizada en el África, en el que se observó que el consumo de alimentos expendidos por ambulantes aumenta el riesgo de presentar dispepsia (19). Este punto mencionado anteriormente debería ampliarse a futuro, así como, ver probables intervenciones para mejorar este problema.

Otro dato relevante fue que quienes omitían regularmente el almuerzo y la cena tenían mayor frecuencia de dispepsia, esto se debe a que los momentos de mayor demanda eran a la hora del almuerzo y la cena, cuyo horario de trabajo es continuo y prevalece principalmente la atención al cliente, lo que hace difícil alimentarse en el almuerzo y la cena. En esta asociación no se ha encontrado bibliografía que apoye esto, por lo que, estos resultados deben ser comprobados, siendo un importante punto de partida para futuras investigaciones, tomados como un "proxy" de la relación que tienen los hábitos alimentarios con esta enfermedad (20).

Una de las principales limitaciones es que nuestro estudio no pudo buscar causas orgánicas como generadoras de la dispepsia, porque fue un estudio basado en una encuesta, pero aún los resultados son importantes debido a la alta sensibilidad y especificidad que ha mostrado esta prueba, ya que es una población que antes casi no ha sido evaluada. Esto sugiere que se deben realizar futuras investigaciones que puedan contar con estudios de endoscopía de vías digestivas altas y con la evaluación individual de cada encuestado.

Con base en los resultados hallados, se puede concluir que se encontró una alta frecuencia de DF en estos empleados; además, tener problemas para conciliar el sueño, consumir alcohol, alimentarse en establecimientos de comida rápida y no almorzar o cenar están asociados a la frecuencia de DF.

Se recomienda hacer estudios en otras poblaciones similares, para, de este modo, poder encontrar otros factores asociados. Todo esto con el fin de motivar investigaciones a futuro, que puedan brindar las medidas de vigilancia y control en estos grupos de riesgo, y de este modo mejorar la calidad de vida de este tipo de población.

\section{REFERENCIAS}

1. Mejía CR, Quezada-Osoria C, Verastegui-Díaz A, Cárdenas MM, García-Moreno KM, Quiñones-Laveriano DM. Factores psicosociales y hábitos asociados con dispepsia funcional en internos de un hospital nacional en Piura, Perú. Rev Col Gastroenterol. 2016;31(4):354-9.
2. Ocampo-Bustos RM, Juárez-García A, Arias-Galicia LF, Hindrichs I. Factores psicosociales asociados a engagement en empleados de un restaurante de Morelos, México. Liberabit. 2015;21(2):207-19.

3. Zolezzi-Francis A. Las enfermedades funcionales gastrointestinales y Roma III. Rev Gastroenterol Perú. 2007; 27(2):177-84.

4. Miwa H, Watari J, Fukui H, Oshima T, Tomita T. Pathogenesis and management of functional dyspepsia. Nippon Rinsho. 2010; 68(7):1391-401.

5. Mahadeva S, Goh KL. Epidemiology of functional dyspepsia: A global perspective. World J Gastroenterol. 2006; 12(17):2661-6.

6. El Serag HB, Talley NJ. Systemic review: The prevalence and clinical course of functional dyspepsia. Aliment Pharmacol Ther. 2004;19(6):643-54.

7. Curioso W, Donaires-Mendoza N, Bacilio-Zerpa C, Ganoza-Gallardo C, León-Barúa R. Prevalencia y asociación de la dispepsia y el síndrome de intestino irritable en una comunidad de la Selva Peruana. Rev Gastroenterology. 2002; 22(2):11.

8. Bisbal-Murrugarra $\mathrm{O}$, León-Barúa $\mathrm{R}$, Berendson-Seminario $\mathrm{R}$, Biber-Poillevard M. A new questionnaire for the diagnosis of dyspepsia. Acta Gastroenterol Latinoam. 2002;32:25-8.

9. Becoña E, Vázquez FL. The Fagerström test for nicotine dependence in a Spanish sample. Psychol Rep. 1998;83:1455-8.

10. Rodríguez-Martos A, Navarro RM, Vecino C, et al. Validación de los cuestionarios KFA (CBA) y CAGE para el diagnóstico del alcoholismo. Drogalcohol. 1986;11:132-9.

11. Miwa H, Ghoshal UC, Fock KM, Gonlachanvit S, Gwee KA, Ang TL, et al. Asian Consensus Report on Functional Dyspepsia. J Neurogastroenterol Motil. 2012;18:150-68.

12. Valenzuela-Narváez DR, Gayoso-Cervantes M. Estrés laboral y su correlación con la prevalencia de dispepsia funcional en militares en actividad que acudieron al hospital geriátrico del ejército. Rev Gastroenterol Perú. 2017;37(1):16-21.

13. Lee SW, Lien HC, Lee TY, Yang SS, Yeh HZ, Chang CS. Etiologies of Dyspepsia among a Chinese Population: One Hospital-Based Study. Open Journal of Gastroenterology. 2014;4:249-54.

14. Yu J, Liu S, Fang XC, Zhang J, Gao J, Xiao YL, et al. Gastrointestinal symptoms and associated factors in Chinese patients with functional dyspepsia. World J Gastroenterol WJG. 2013;19:5357-64.

15. Talledo-Ulfe L, Buitrago OD, Filorio Y, Casanova F, Campos L, Cortés F, et al. Factores asociados a dispepsia no investigada en estudiantes de 4 facultades de medicina de Latinoamérica: estudio multicéntrico. Revista de Gastroenterología de México. 2018;83(3):215-22.

16. Lacy BE, Everhart K, Crowell MD. Functional Dyspepsia is associated with sleep disorders. Clin Gastroenterol Hepatol. 2011;9:410-4.

17. Molano J, Piñeros S, López C. Factores emocionales e impacto sobre la escolaridad y actividad social en adoles- 
centes con dispepsia y reflujo gastroesofágico. Rev Colomb Gastroenterol. 2009;24(4):364-72.

18. Vargas M, Talledo UL, Samaniego RO, Heredia P, Rodríguez CAS, Mogollón CA, et al. Dispepsia funcional en estudiantes de ocho facultades de medicina peruanas. Influencia de los hábitos. Acta Gastroenterol Latinoam. 2016;46(2):95-101.

19. Barker SF, Amoah P, Drechsel P. A probabilistic model of gastroenteritis risks associated with consumption of street food salads in Kumasi, Ghana. Evaluation of methods to estimate pathogen dose from water, produce or food quality. Sci Total Environ. 2014;487:130-42.

20. Benites-Velásquez BB, Bellido-Boza LE. Asociación de la dispepsia funcional con los factores psicológicos y los hábitos alimentarios en estudiantes de la Facultad de Medicina de la UNMSM, Lima-Perú. UNMSM [internet] 2006 [acceso el 7 de diciembre de 2018]. Disponible en: http:// cybertesis.unmsm.edu.pe/handle/cybertesis/772. 